\title{
The use of an endonasal Doppler to locate the vascular pedicle of a nasoseptal flap prior to harvesting in patients who had previous irradiation to the nasopharynx
}

\author{
Samuel MW Chow, Michael CF Tong \\ Prince of Wales Hospital, Hong Kong
}

Aim:

To assess the survival rates of nasoseptal flaps (NSF) harvested with and without the use of an endonasal Doppler (Minidop Detector, Koven ${ }^{\circledR}$, USA) to locate the vascular pedicle in patients who had previous irradiation to the nasopharynx (NP).

Method:

Retrospective chart review

Results:

Between May 2012 and Jan 2018, a NSF was used to cover the nasopharyngeal surgical wound in 16 patients who had previous irradiation to the NP in a tertiary referral centre. 10 cases had endoscopic nasopharyngectomies for recurrent T1 nasopharyngeal carcinoma (NPC), 3 cases underwent combined maxillary swing and endoscopic nasopharyngectomies for recurrent T1 NPC, 1 cases had revision flap surgery for NSF failure, 1 case had endoscopic debridement for nasopharyngeal soft tissue necrosis, 1 had transclival resection of a recurrent clival chordoma. Endonasal Doppler was not used to locate the vascular pedicle of NSF before harvesting in 9 cases, and 4 out of 9 flaps survived (44\%). Endonasal Doppler was used to locate the vascular pedicle with the aim to protect it during harvesting in 7 cases. A positive identification was achieved in 5 cases, and all 5 flaps survived (100\%). The pedicle could not be located in 2 out of 7 cases. As a result, a NSF with wide pedicle based from the sphenoid ostium to the roof of the posterior choana was harvested, but eventually the distal end of the flaps developed ischaemia. The Pearson Chi-Square Test of Independence showed statistically significant better NSF survival rates in patients who had a positive identification of the vascular pedicle using the endonasal Doppler $(\mathrm{p}=0.017)$.

Conclusion:

Radiotherapy can cause stenosis or occlusion of the posterior septal branch of sphenopalatine artery. Using an endonasal Doppler to locate the vascular pedicle of a NSF harvested to cover a nasopharyngeal wound in patients who had previous irradiation to the NP increases the survival rate. Positive localization of the pedicle helps surgeon to protect the pedicle during harvesting and a negative Doppler should alert surgeon to consider other reconstructive options. 